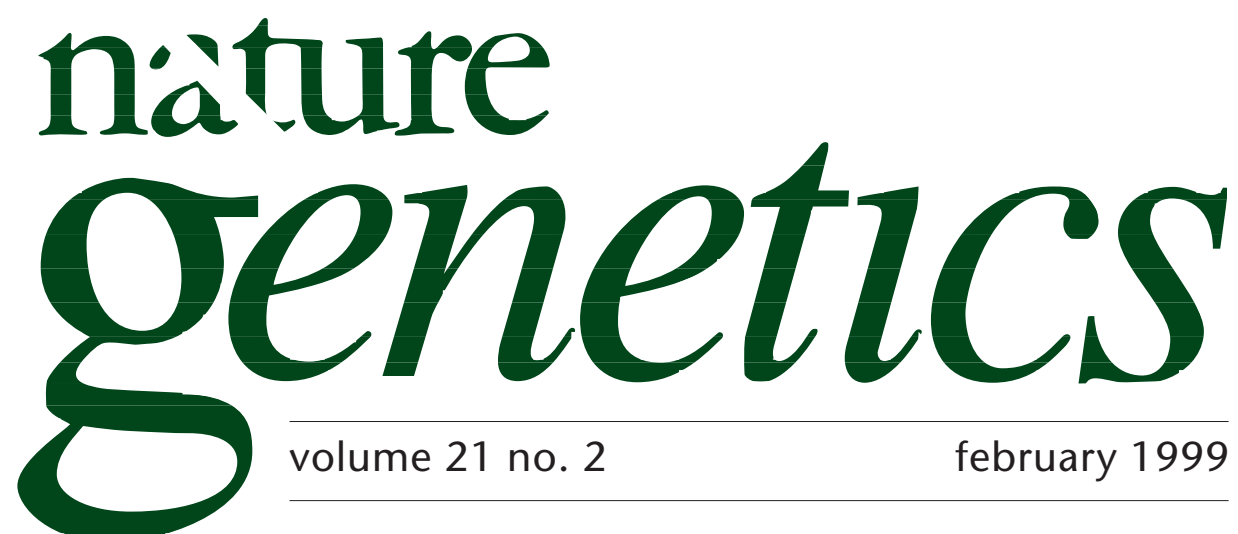

\title{
Patenting ESTs: is it worth it?
}

As the completed sequence of the human genome flows into public databases, those hoping to stake a primary claim on a DNA sequence will already have their patent application in queue at the United States Patent and Trademark Office

(USPTO). The issue of the first patent for an expressed sequence tag

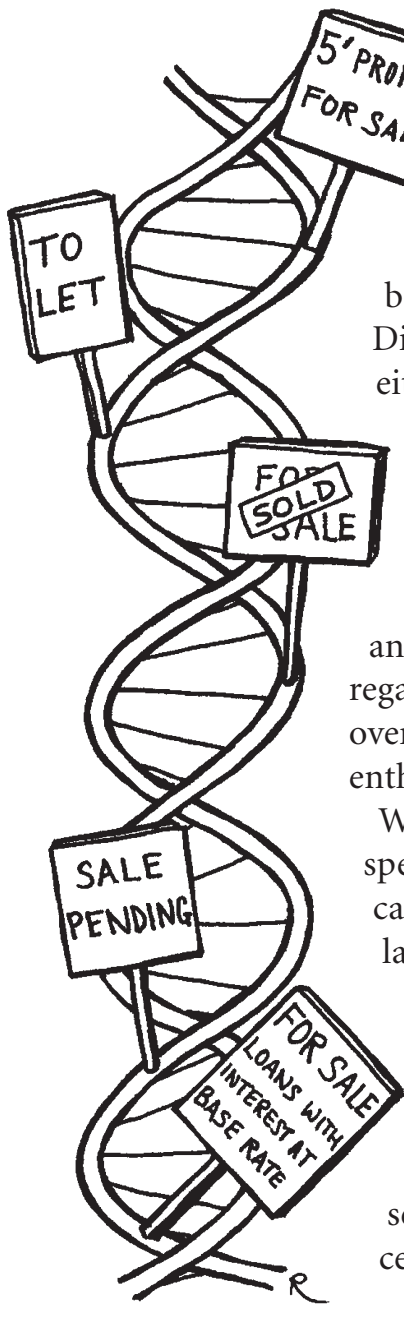

REAL ESTATE VALUES

(EST) to Incyte Pharmaceuticals, Inc., on 6 October last year was no doubt an encouraging sign for the viability of EST patents in the genome market. Delays in patent issuance, the costs and the uncertainty regarding the strength of potential patent claims, however, call into question whether EST patents will prove fruitful.

From a global perspective, the wave of EST patent claims is likely to break only on US shores. The draft of the European Union Biotechnology Directive stipulates that an EST, while not unpatentable a priori, is unlikely to either qualify as an 'inventive step' or be of sufficient industrial utility to be deemed patentable. Although the European Patent Office (EPO) has yet to decide on any EST application, it shares the Directive's view that ESTs are unlikely to be patentable. The EPO and the Japanese Patent Office (JPO) have been slow to establish a definitive policy on EST patentability, largely as a consequence of the relatively few EST patent applications filed. The EPO and JPO, unlike the USPTO, publish patent applications 18 months after filing, regardless of whether the patent has been issued. Together with the uncertainty over the patent eligibility of ESTs in these countries, this may have dampened the enthusiasm of potential applicants.

While EST patentablity per se is no longer in question in the US, there is speculation regarding the scope of an EST patent claim. The scope is implicated by the wording of a claim, which can employ either 'open' or 'closed' language. Closed language, such as ". . a DNA sequence consisting of ...", restricts the claim scope to only the disclosed sequence. Open language, such as "... a DNA sequence comprising...", extends it to include unknown sequences that contain the disclosed sequence. The EST patent issued to Incyte contains 'open' claim language and therefore has the potential to extend to all as yet unknown genes containing the sequences disclosed. The National Institutes of Health (NIH), US, is concerned that broad EST claims may jeopardize the research and commercial development of full-length sequences and has urged the USPTO to limit the scope of EST patents to the disclosed sequence, particularly for those for which the primary utility is as a research tool. 
On the other hand, John Doll, Director of Biotechnology Patent Examination, USPTO, maintains the view "that if an applicant files a new DNA sequence, for which there is nothing to suggest or render it obvious, then the applicant should be allowed the full scope of the broad protection." Even an EST patent of broad scope, however, will not preclude patenting of longer or full-length coding sequences discovered later. US patent law defines gene sequences by their specific structural characteristics; knowledge of sequences significantly extending either end of a disclosed EST sequence is therefore considered neither obvious nor anticipated and thus patentable. It is possible that several patents will be awarded for different and/or overlapping segments of the same gene. Each EST patent with broad claim scope will "dominate" and, as a consequence, the commercial application of an EST might infringe upon the patent claims of another EST for the same gene. Inevitably, commercial development of the full-length gene will require negotiating the cumulative stacking of royalties owed to the various EST patent holders. These overlapping intellectual properties will add a new layer of complexity to the commercialization of at least some DNA 'inventions' in the US.

Many patent applications for ESTs claim utility as a research tool. The potential value of such patent claims will depend on whether the patent is issued within a sufficient time frame to allow exploitation before the sequence information reaches the public domain via global sequencing efforts. The USPTO takes, on average, 24 to 26 months to issue a biotechnology patent. Although tens of thousands of EST sequences can be filed in a single application - all of them retaining the original filing date- the USPTO restricts its examination to ten sequences per application at a time. The applicant does have the option of filing additional groups of ten sequences to be examined simultaneously. Nevertheless, for an application containing 10,000 ESTs, a considerable period of time will elapse before many of them see the light of day as patented entities. Given the rapid rate at which sequence information is becoming publicly available, it is quite possible that the research community will have accessed the sequence information, mined it for any potential functional utility and progressed to a stage of development beyond the scope of the EST patent claim before the patent is issued. Patenting is a costly process, involving a minimum of $\$ 1,000$ dollars per 10 sequences in USPTO fees for filing and issuance of the patent - which is a miniscule fraction of the attorney fees. At some point, companies such as Incyte and Human Genome Sciences will have to re-evaluate their extensive portfolio of EST patent applications to ascertain whether it is in their economic interests to proceed with prosecution of all ESTs filed.

Irrespective of the breadth of patents issued by the USPTO, it is the courts that will decide the validity and scope of claims. Colin Sandercock of Foley \& Lardner, a law firm specializing in biotechnology patents, believes that "while it is difficult right now to predict what the scope of EST patent claims will be, the current judicial climate suggests that it will likely be relatively narrow." The courts consider several factors when deciding claim scope, including whether the patent discloses sufficient information for someone 'skilled in the art' at the time the patent application was filed to make full use of the scope of the invention. The extent of additional experimentation required to develop the commercial utility of the patented entity may also be taken into account. Thus, until ESTs have had their day in court - which Sandercock predicts may not be for a decade or so- the boundaries of EST patent claims will remain ill defined.

Obtaining a broad patent claim on an EST raises expectations in the eyes of would-be patentees. For a lucky few, an EST patent may be the pot of gold at the end of the genomic rainbow. However, for the vast majority, they are likely to be a sunk investment, issued too late and of limited claim on downstream applications.

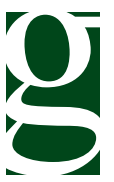

